\title{
Observed Area Under the Urinary Excretion Rate Curve infinity
}

National Cancer Institute

\section{Source}

National Cancer Institute. Observed Area Under the Urinary Excretion Rate Curve infinity. NCI Thesaurus. Code C85767.

The area under the urinary excretion rate curve (AURC) extrapolated to infinity, based on the last observed excretion rate. 